\title{
VIBRATIONS DETECTION IN INDUSTRIAL PUMPS BASED ON SPECTRAL ANALYSIS TO INCREASE THEIR EFFICIENCY
}

\author{
Belhadef RACHID \\ University of Sedik Ben yahia of Jijel \\ Ahmed HAFAIFA \\ University of Djelfa \\ Mohamed BOUMEHRAZ \\ University of Biskra
}

\begin{abstract}
:
Spectral analysis is the key tool for the study of vibration signals in rotating machinery. In this work, the vibration analysis applied for conditional preventive maintenance of such machines is proposed, as part of resolved problems related to vibration detection on the organs of these machines. The vibration signal of a centrifugal pump was treated to mount the benefits of the approach proposed. The obtained results present the signal estimation of a pump vibration using Fourier transform technique compared by the spectral analysis methods based on Prony approach.
\end{abstract}

Key words: Spectral analysis, vibration signal, vibration analysis, rotating machinery, centrifugal pump, signal estimation, Fourier transform, Prony approach

\section{INTRODUCTION}

Rotating machines are massively present in many industrial plants. However, these rotating machines encounter many failures. For this it is necessary to have some oversight mechanisms, to learn about their state of operation of these machines, in order to take timely decisions about their operating state. A means for monitoring the operating state of a rotating machine is the collection and analysis of vibration $[1,2,3,4]$. The sensed vibrations are collected and analyzed information on the state of a system, particularly on the state of a revolving thing, or elements thereof vibration can then be viewed as a symptom. Thus treatment of the sensed vibration signals (signatures) used to define a normal or abnormal state of the machine or machine part and with an archive device allow the detection or diagnosis of the machine. For an interested note, the acquisition of these signatures can be done without having to stop the machine.

Indeed, the spectral analysis is based on a systematic analysis of the vibration signal for the presence of vibratory images of all defects that can affect the installation considered $[5,6,7,10,11]$. This requires knowledge of the kinematic characteristics of the various component parts, their rotational speed or more preferably their frequency examined motion system. In this work, methods of estimating the spectral density are proposed; these methods are good spectral analysis tools, given by the calculation point of view and allow obtaining good results for a large class of signals.

In this work, the normal operation of a rotating machine is examined, through the example of a centrifugal pump system, the vas kind of the reporting pump defects are pre- sent and correct to show the effectiveness of the proposed approach based on the spectral analysis.

\section{SPECTRAL ANALYSIS}

The first way of representation of the vibration signal output from a sensor, the representation is a function of time (time representation). This representation is used to monitor the vibration behavior of a machine in accordance with these operating parameters. Actually, there are numerous power spectral density estimation methods; it is assumed that they can be classified into two major families of non-parametric methods and parametric methods $[5,9$, 11]. In this work, parametric methods are used for the estimate of the spectral density of the examined pump.

\section{Parametric modeling}

Parametric modeling allows representing all the spectral information by a small number of parameters, for the autoregressive modeling in adjusted mean, most processes are well approximated by a linear rational model. A general ARMA model (Auto Regressive Moving Average) is defined by the recurrence function given by:

$$
x(n)=-\sum_{k=1}^{p} a_{k} x(n-k)+\sum_{k=0}^{q} b_{k} u(n-k)
$$

where:

$a_{k}$ is the parameters of the autoregressive part (AR), $b_{k}$ is the parameters of the autoregressive part (MA), $P$ is the order of the part $A R$, $q$ is the order of the party MA, with $\mathrm{a}_{0}=\mathrm{b}_{0}=1$ 
The function (1) represents an output of a linear filter, whose input is $u(n)$ and the transfer function such that:

$$
H(z)=\frac{X(z)}{U(z)}=\frac{1+\sum_{i=1}^{q} b_{i} z^{-i}}{1+\sum_{i=1}^{p} a_{i} z^{-i}}=\frac{B(z)}{A(z)}
$$

With:

$$
A(z)=1+\sum_{k=1}^{p} a_{k} z^{-k}
$$

And:

$$
B(z)=1+\sum_{k=1}^{q} b_{k} z^{-k}
$$

$B(z)$ and $A(z)$ is the $Z$ transform of the branch,

$\mathrm{H}(\mathrm{z})$ represents the transfer function pole/zero model.

It is shown that to ensure that the process $x(n)$ is stationary, it is necessary that $\mathrm{H}(\mathrm{z})$ is stable and causal, and that $\mathrm{A}(\mathrm{z})$ has its zeros inside the unit circle. It is considered that the input $u(n)$ of the filter is white noise and it is also assumed in general that $u(n)$ is a Gaussian distribution. In case the parameters for this AR zero autoregressive modeling with $1 \leq i \leq q$ is simple, which gives:

$$
x(n)=-\sum_{i=1}^{p} a_{i} x(n-i)+u(n)
$$

Thus, the polynomial $B(z)$ reduces to a constant $B(z)=1$, and the transfer function $\mathrm{H}(\mathrm{z})$ then only contains the poles. For this reason, this model is also called an all-pole model

$$
A R(p): x(n)=-\sum_{k=1}^{p} a_{k} x(n-k)+u(n)
$$

The modeling adjusted average, for the parameters are zero for $1 \leq \mathrm{i} \leq \mathrm{q}$, giving:

$$
x(n)=-\sum_{i=1}^{p} b_{i} x(n-i)+u(n)
$$

The corresponding transfer function is $\mathrm{H}(\mathrm{z})=\mathrm{B}(\mathrm{z})$, it is characterized by a polynomial having only zeros, which defines an all-zero model.

$$
M A(p): x(n)=-\sum_{k=1}^{q} b_{k} x(n-k)
$$

The power spectral density is the $Z$ transform of the correlation function. For the ARMA $(p, q)$ we get:

$$
\begin{gathered}
S_{x}(z)=H(z) H^{*}\left(\frac{1}{z^{*}}\right) S_{u}(z) \\
S_{x}(z)=\frac{B(z) B^{*}\left(\frac{1}{z^{*}}\right)}{A(z) A^{*}\left(\frac{1}{z^{*}}\right)} S_{u}(z)
\end{gathered}
$$

Appoints three reasons, at least we incident to estimate the spectral density of a process by performing an autoregressive modeling. The spectral density of the autoregressive $x(n)$ is given by:

$$
s_{X}(z)=E\left\{X(z) \cdot X^{*}(z)\right\}
$$

Where the ${ }^{*}$ indicates the amount combined is the expectation operator, $\mathrm{E}$ is the expectation operator and $\mathrm{X}(\mathrm{z})$ is the Z-transform of $x$ process.

Since $x(n)$ is the output of an all-pole filter transfer function $1 / A(z)$ and input $u(n)$, it can be expressed in the complex domain:

$$
X(z)=H(z) \cdot U(z)=\frac{1}{A(z)} U(z)
$$

However, $U(n)$ is stationary white noise with zero mean and variance $\delta^{2}$, therefore the signal will also be generated if the stationary transfer function filter is stable, that is to say if replacing the poles of this function transfer $1 / A(z)$ is located at the inside of the unit circle.

By replacing $X(z)$ by its expression, becomes:

$$
S_{x}(z)=\frac{1}{|A(z)|^{2}} E\left\{U(z) \cdot U^{*}(z)\right\}
$$

The between being a white noise, we get :

$$
E\left\{U(z) \cdot U^{*}(z)\right\}=S_{u}(z)=\sigma^{2}
$$

Finally, according to (10) the spectral density of a process $A R \times(n)$ is expressed as AR (p)

$$
S_{x}(z)=\frac{\sigma^{2}}{|A(z)|^{2}}=\frac{\sigma^{2}}{\left|1+\sum_{k=1}^{p} a_{k} e^{-j 2 \pi k f}\right|^{2}}
$$

With:

$$
z=e^{2 \pi j f}
$$

Or $f$ is set to reduce the frequency interval: $-1 / 2 \leq f \leq 1 / 2$

Therefore, estimating the spectral density of an AR process returns to calculate AR coefficients $a_{i}$.

In practical reality, there are very few algorithms that have been developed for spectral power density estimation ARMA because of the nonlinearity of the moving average part. One can define a signal $y(n)$ have an ARMA model by:

$$
y(n)=\sum_{i=0}^{q} b(i) e(n-i)-\sum_{k=1}^{p} a(k) y(n-k)
$$

Once we identify the parameter of the ARMA $(p, q)$, spectral estimation defined by:

$$
P_{A R M A}(f)=|H(\exp (2 \pi j f \Delta t))|^{2}
$$




$$
P_{A R M A}(f)=\frac{\sigma^{2} \Delta t\left|1+\sum_{i=0}^{q} b(i) \exp (-2 \pi j f i \Delta t)\right|^{2}}{\left|1+\sum_{k=1}^{p} a(k) \exp (-2 \pi j f k \Delta t)\right|^{2}}
$$

The relationship between the ARMA parameters and the autocorrelation function is easily obtained by multiplying equation (14) by $y(n-1)$, and taking the expected value of the expression:

$$
\begin{gathered}
R_{y y}(n)=\sum_{i=0}^{q} b(k) R_{\xi y}(i-k)-\sum_{k=1}^{p} a(k) R_{y y}(i-k) \\
R \xi y=E[\xi(n) y(n-k)]
\end{gathered}
$$

With $\mathrm{R}_{\xi y}$ for $\mathrm{k} \succ 0$, in practice $\mathrm{p}_{\mathrm{k}}$ is always positive or zero, that is to say the denominator and the numerator higher than that (causal system).

$r_{y y}(i)=\left\{\begin{array}{l}-\sum_{k=1}^{p} a(k) r_{y y}(i-k)+\sum_{k=0}^{q} b(i) R_{\xi y}(i-k) \\ -\sum_{k=1}^{p} a(k) r_{y y}(i-k)\end{array}\right.$

$$
\begin{aligned}
& \text { for } i=1,2,3 \ldots \ldots \ldots . q \\
& \text { for } i=q+1 ; q+2, \ldots \ldots
\end{aligned}
$$

To achieve the equations YULE WALKER is shown that $R_{\xi y}=\sigma^{2} h^{*}-k$. This gives the flowing formula:

$r_{y y}(i)=\left\{\begin{array}{l}-\sum_{k=1}^{p} a(k) r_{y y}(i-k)+\sigma^{2} \sum_{k=0}^{q} b(i) R_{\xi y}(i-k) \\ -\sum_{k=1}^{p} a(k) r_{y y}(i-k)\end{array}\right.$

$$
\text { for } i=1,2,3 \ldots \ldots \ldots q
$$

$$
\text { for } i=q+1 ; q+2, \ldots \ldots
$$

These equations in an ARMA process are similar to those for a Yule Walker AR process, these equations can be rewritten in matrix form as follows:

$$
\left[\begin{array}{llll}
r_{y y}(q) & r_{y y}(q-1) & \ldots & r_{y y}(q-p+1) \\
r_{y y}(q+1) & r_{y y}(q) & & r_{y y}(q-p+2) \\
\vdots & \vdots & \vdots & \\
r_{y y}(q+p-1) & r_{y y}(q+p-2) & \ldots & r_{y y}(q)
\end{array}\right]=
$$

$$
=\left[\begin{array}{l}
a(1) \\
a(2) \\
\vdots \\
a(p)
\end{array}\right]=\left[\begin{array}{l}
r_{y y}(q+1) \\
r_{y y}(q+2) \\
\vdots \\
r_{y y}(q+p)
\end{array}\right]
$$

This relationship is known as scopes equations or modified Yule WQLKER, the matrix $R_{y y}$ is not symmetrical, to solve this system of equations (18). Once the parameters calculated AR, MA parameters are determined by data filtering with a filter whose transfer function is the following:

$$
\hat{A}(z)=1+\sum_{k=1}^{p} a z^{-k}
$$

Then we have the equivalence:

$$
\frac{B(z)}{A(z)} \hat{A}(z)=B(z)
$$

The filtered sequence NP in length is obtained by the following convolution equation:

$$
F(n)=x(n)+\sum_{m=1}^{p} a(m) x(n-m)
$$

To determine the power spectral density, only one needs to estimate $\Delta t \sigma^{2}|\mathrm{~B}(\mathrm{f})|^{2}$ this is to say the numerator. In this case the MA parameters are not requests. One can use the correlogram method for estimating the power spectral density MA:

$$
\begin{gathered}
\Delta t \sigma^{2}|\hat{B}(f)|^{2}=\sum_{m=-p}^{q} \hat{r_{y y}} \exp (-2 \pi j f m \Delta t) \\
\hat{R} f f(m)=\sum_{m=-p}^{q} a(n) \hat{r} y y(m-n)
\end{gathered}
$$

Finally we calculate the power spectral density of ARMA:

$P_{A R M A}=\frac{\sum_{m=-q}^{q} \hat{R}(m) \exp (-2 \pi j f m \Delta t}{|A \exp (-2 \pi j f \Delta t)|}$

For the parameter estimation of an AR model, we seek to find the best prediction order $p$ of $x(n)$ :

$$
\hat{x}(n)=-\sum_{k=1}^{p} a_{k} x(n-k)
$$


For this we look for the coefficients that minimize the power of the prediction error:

$$
\rho=\mathrm{E}\left\{\mid e\left(\left.n\right|^{2}\right\}=\mathrm{E}\left\{\hat{x}|(n)-x(n)|^{2}\right\}\right.
$$

With:

$$
\begin{aligned}
& r=\left(\begin{array}{c}
r_{x x}(1) \\
r_{x x}(2) \\
\cdot \\
\cdot \\
r_{x x}(p)
\end{array}\right) \quad \alpha=\left(\begin{array}{l}
\alpha_{1} \\
\alpha_{2} \\
\cdot \\
\cdot \\
\alpha_{p}
\end{array}\right) \\
& R=\left[\begin{array}{ccccc}
r_{x x}(0) & r_{x x}(-1) & \ldots & r_{x x}(-p+1) \\
r_{x x}(1) & r_{x x}(0) & & r_{x x}(-p+2) \\
\vdots & \ddots & \vdots & \\
r_{x x}(p-1) & r_{x x}(p-2) & \ldots & r_{x x}(0)
\end{array}\right]
\end{aligned}
$$

To estimate of the spectral density by the methods of PRONY, we used the model (26) to approximate the $x(n)$ signal.

$$
x(n)=s(n)=\sum_{m=1}^{p} B_{m} z_{m}^{n}
$$

This model are carriers of four types of information, familiar in signal processing and system modelling: $B_{m}=A_{m} e^{j \phi m}$ et $Z_{m}=e^{i(a m+e n f m) \Delta t}$

Or $A_{m}, \phi_{m}$ are respectively the amplitude, phase, and vector and the damping. The are the frequency and the sampling. For the estimation of polynomial coefficients and the model order, use one of the algorithms for estimating AR parameters. In our work we use the recursive least squares algorithm for. To determine the frequencies and damping coefficients the following equations are used:

$$
\begin{gathered}
a_{i}=\frac{\ln \left|Z_{i}\right|}{\Delta t} \\
f_{i}=\operatorname{tg}^{-1}\left(\frac{\operatorname{Im}\left(Z_{i}\right) / \operatorname{Re} a\left(Z_{i}\right)}{2 \pi \Delta t}\right)
\end{gathered}
$$

To determining amplitudes and phases based on the equations (26) and (27), we used the relation given in (28):

$$
B=\left[{ }_{\phi}^{H} \phi\right]^{-1} \phi^{H} X
$$

The approximate function becomes:

$\hat{x}(n)=\sum_{i=1}^{p} A_{i} \exp \left(\alpha_{i} i \Delta t\right) \exp \left[j\left(2 \pi f_{i} i \Delta t+\phi_{i}\right)\right]$ by:

The power spectral density by the Prony model is given

$$
\left\{\begin{array}{l}
S_{\text {prony }}=|x \hat{(f)}| \\
\hat{x}(f)=\sum_{k=1}^{p} A_{i} \exp \left(j \Phi_{i}\right) \frac{2 \alpha_{i}}{\alpha_{i}^{2}+\left[2 \pi\left(f-f_{i}\right)\right]^{2}}
\end{array}\right.
$$

The signal parametric modeling is a widely used to represent a cutting means, to a level of synthesis and by prior analysis or physical phenomena giving rise to a signal either of the signal generation mechanisms (e.g. filtering, modulation, etc.). It allows to translate, with a low-typically finite number of parameters the main characteristics of the signal. It therefore has the main advantage of reducing the space of representation, in general, to reduce the computational load associated with signal processing. It should be noted that such an approach is feasible only when has many a priori information on the signal. Therefore, the operator must take into account the validity of assumptions since they determine the performance but also the estimation robustness. In the next section we will introduce the fundamental notion on the diagnosis of complex systems.

\section{INDUSTRIAL APPLICATIONS}

The studied machine in this work is a vertical centrifugal pump type, the pump is one of a battery of three pumps that are called "charge pumps" are used to extract LPG (gas, oil liquefies) stored in the buffer storage spheres to a 8 bar pressure and discharging it to the processing unit to a pressure 20 bars, shown in Figure 1.

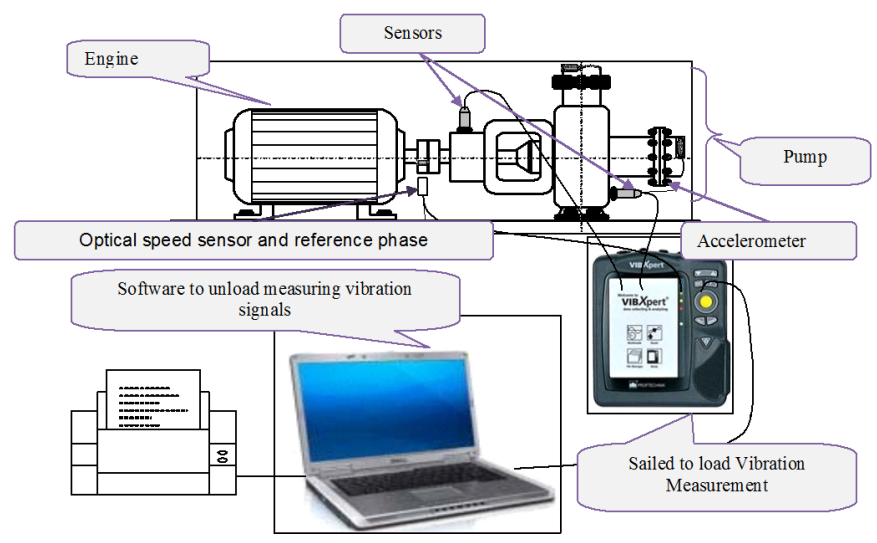

Fig. 1 Vibration detection system applied to a pump

The signal sensed by an optical speed sensor and phase reference placed on overcomes the pump. A record of the vibratory signals emitted by the system has been carried out every day lasting 12 days. Measuring the lay once daily. Each record contains 4069 samples per day with a sampling frequency of $40 \mathrm{kHz}$. The treatment is done for $800 \mathrm{sam}$ ples. The temporal representation of the emitted signal by the system studied are given in the following Figures $2 a, 2 b$, $2 \mathrm{c}$ and $2 \mathrm{~d}$.

We note that the graphs maintain a normal step until 2nd day of vibrations occurring every day until the 9th day. The system will return to the normal state until the jour10ieme. From that day the vibration producing up to 12 th day. The day that the machine was stopped walked. 
a)

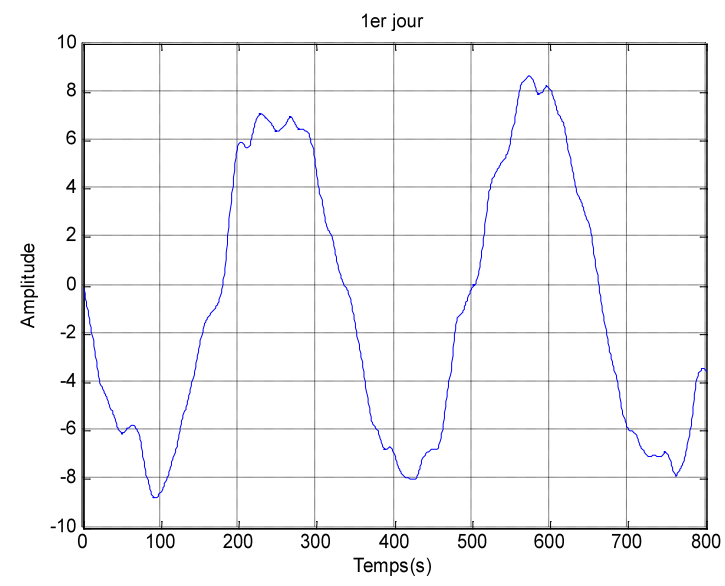

c)

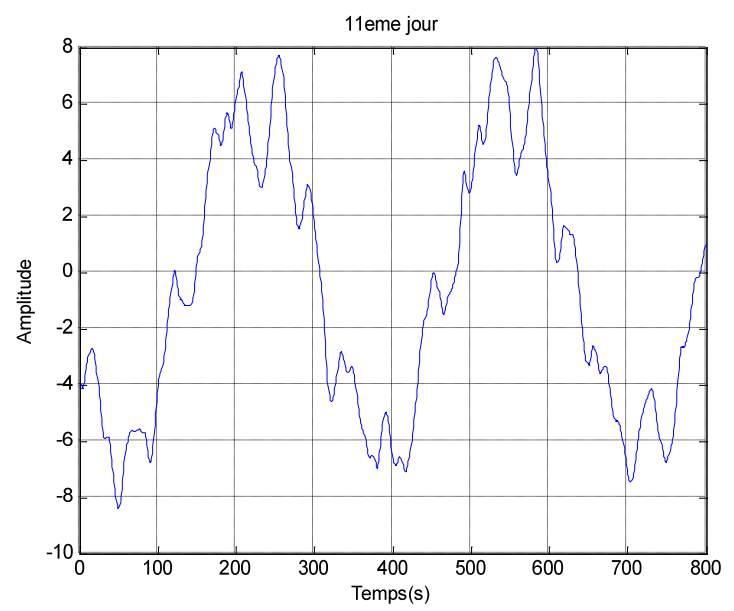

Fig. 2 Emitted signal by the system examines

a)

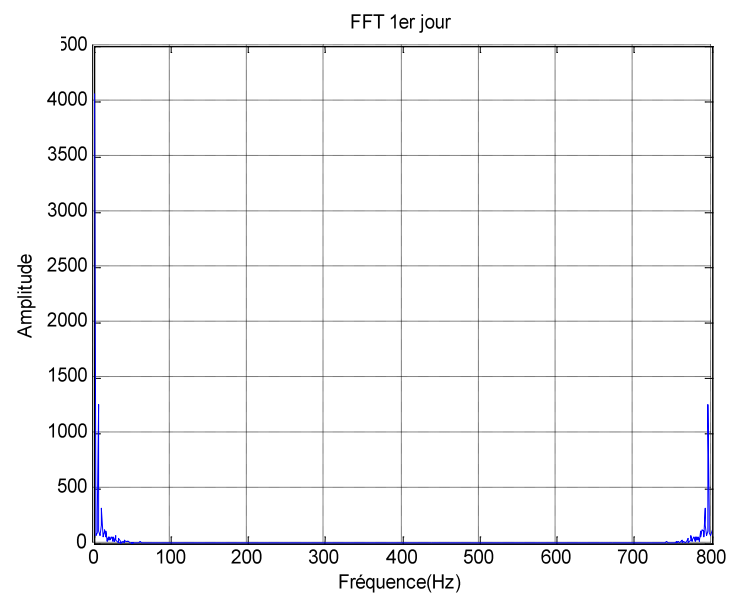

c)

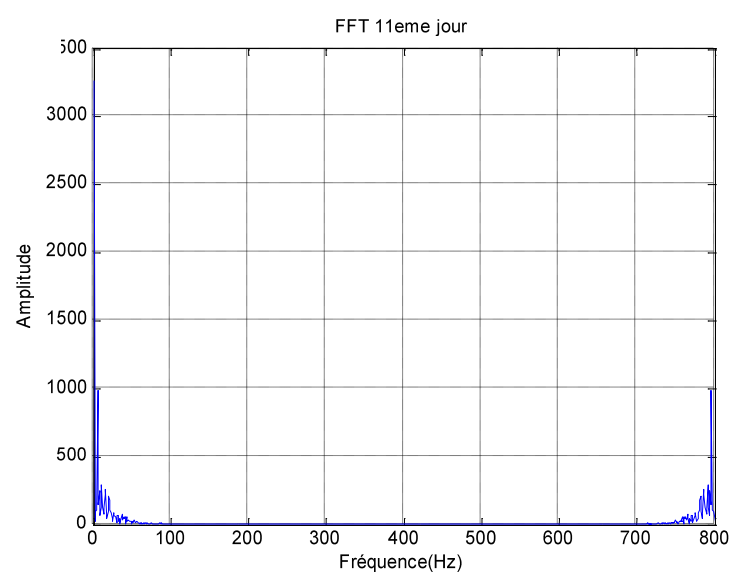

b)

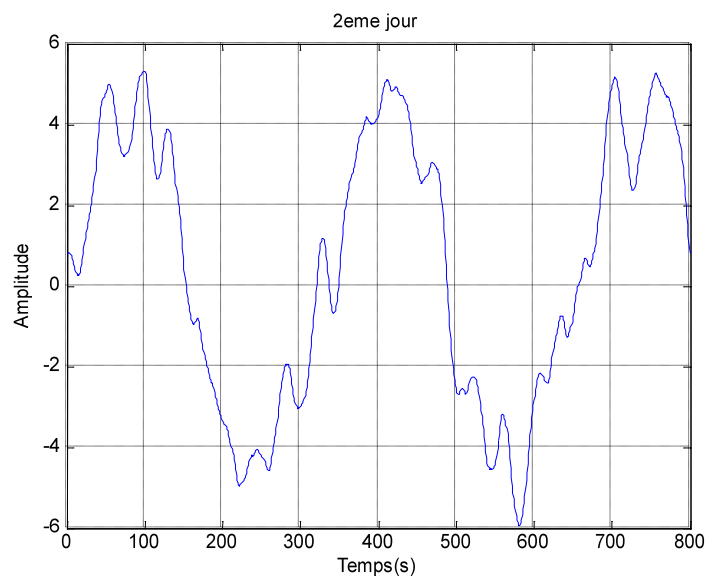

d)

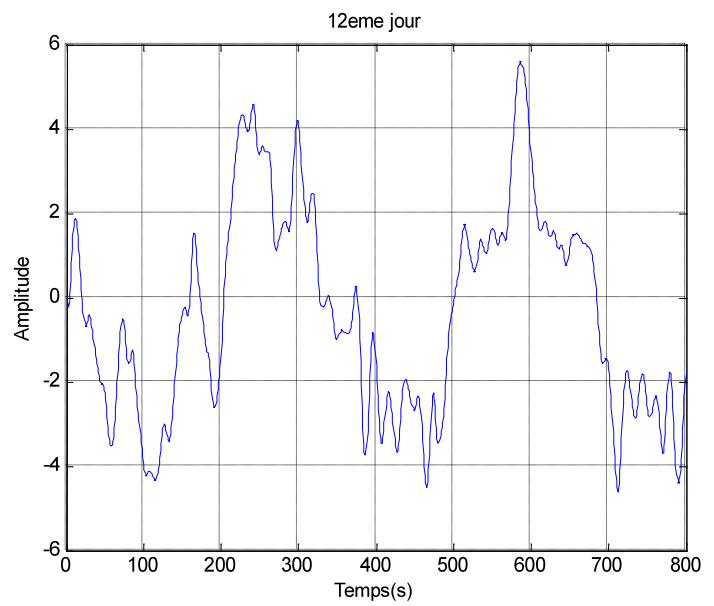

b)

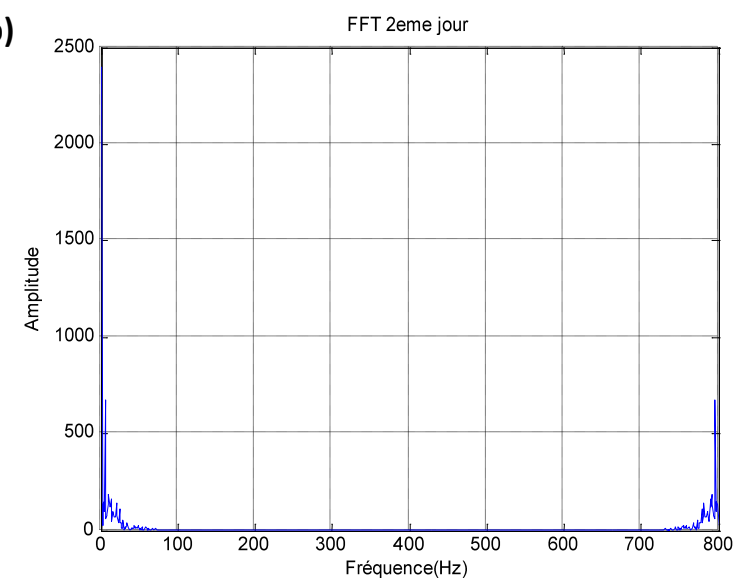

d)

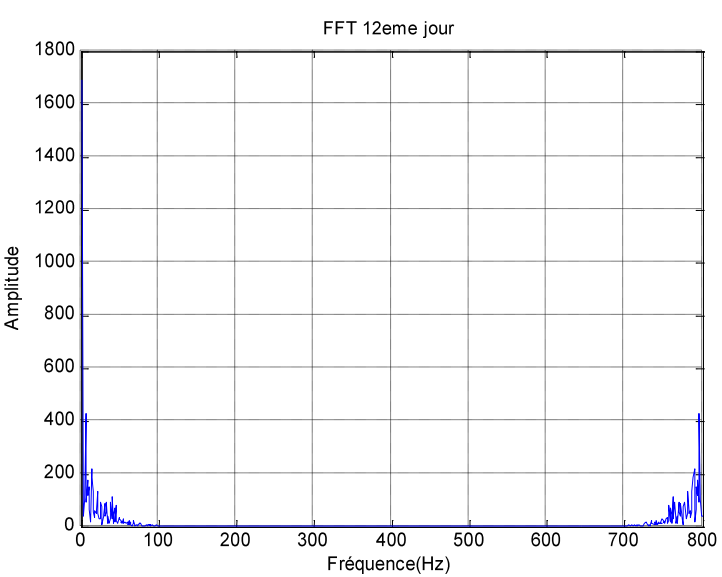


a)

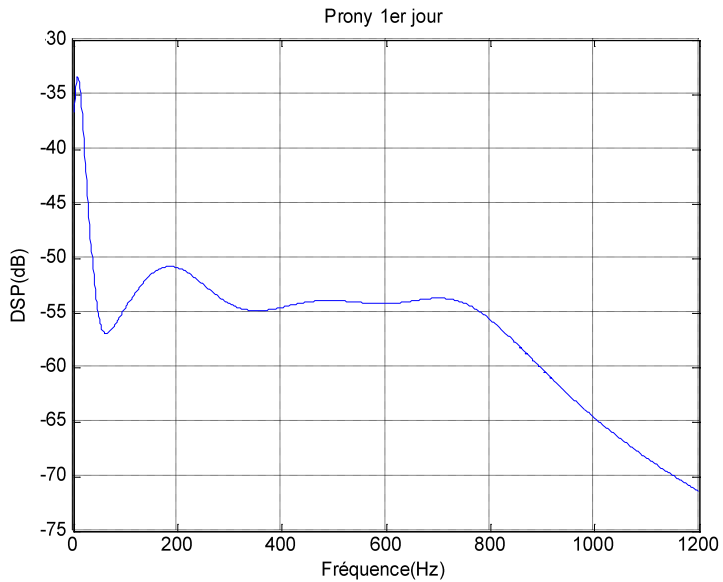

c)

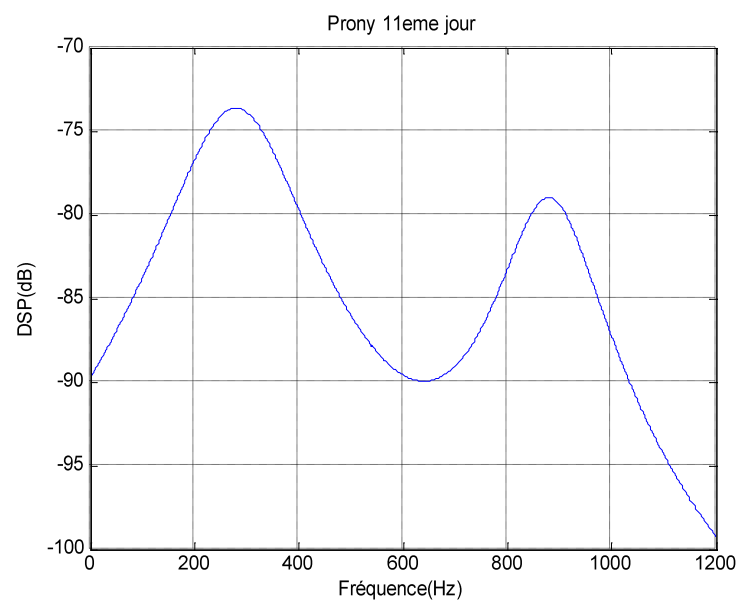

b)

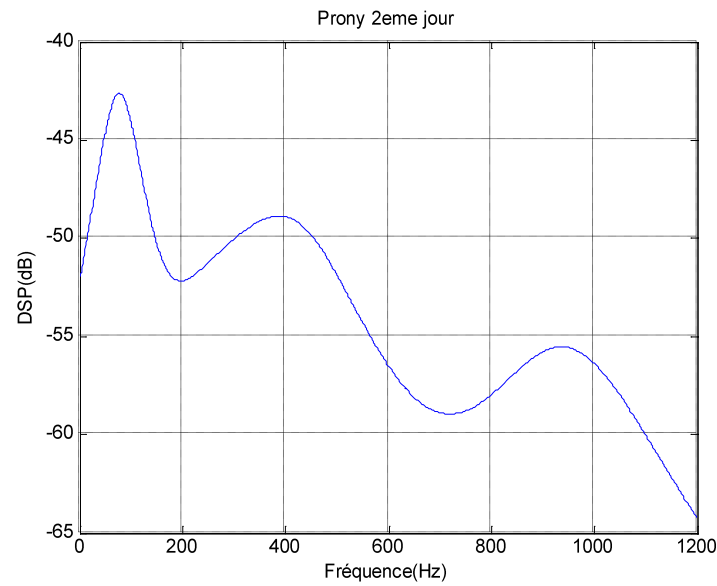

d)

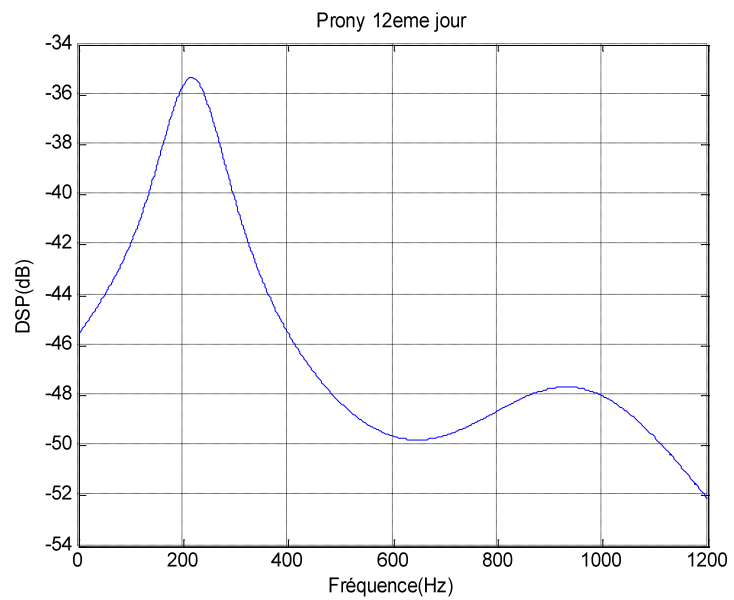

Fig. 4 Obtained spectrum using Prony method

For the presentation of the signal power spectrum emitted by the system studied by the method of FFT, the results are give in the following Figures $3 a, 3 b, 3 c$ and $3 d$.

In note from Figure 3 the presence of a relatively small change in the spectrum of the first day to 5th day presence of fluctuation around main peak at the frequency segmentation of its relative amplitude. From that day to note that the graph it stabilized until the 10th day, the presence of fluctuation around the main peak at the frequency segmentation of its relative magnitude. And finally, for the presentation of the emitted signals by the system power spectrum by using Prony method is shown in following Figures $4 a, 4 b$, $4 \mathrm{c}$ and $4 \mathrm{~d}$.

The Figure 4 shows the spectra of the vibrations of the pump by the Prony method, the power spectra, in this results two peaks which correspond to the frequencies of the multiplied frequency of the vibrations. From the first day to the second day, from the third day we see a displacement of pic1 and pic2 and an increase in the relative amplitude of the peak 1 on the fourth day.

\section{CONCLUSION}

This work shown that the spectral analysis method allows an unbiased estimate of the frequency of a signal in the presence of noise in a stationary or non-stationary environment and the algorithms quickly enough to track the evolution thereof over time with accuracy. Also the studied approach based on probability monitor and diagnose, give the most responded temporal signals and see the Fast Fourier Transform methods of spectral analysis compared by Prony method. The obtained results clearly show the pres- ence of defects on the third day, it is remarkable that the displacement of the two peak 1 and 2 and increased the relative amplitude in the examined pump.

\section{REFERENCES}

[1] Cl. Buerhop, D. Schlegel, M. Niess, C. Vodermayer, R. Weißmann and C.J. Brabec. "Reliability of IRimaging of PV-plants under operating conditions", in Solar Energy Materials and Solar Cells, vol. 107, December 2012, pp. 154-164.

[2] M.J. Chevalier, D.J. Smith and D.W. Dean. „The reliability of structural systems operating at high temperature: Replacing engineering judgement with operational experience", in International Journal of Pressure Vessels and Piping, vol. 98, October 2012, pp. 65-75.

[3] C. Bunea, T.A. Mazzuchi, S. Sarkani and H. Chang. „Application of modern reliability database techniques to military system data", in Reliability Engineering \& System Safety, vol. 93(1), January 2008, pp. 14-27.

[4] S. Eisinger and U.K. Rakowsky. „Modeling of uncertainties in reliability centered maintenance - a probabilistic approach", in Reliability Engineering \& System Safety, vol. 71(2), February 2001, pp. 159-164.

[5] M. Gaceb, R. Bouzid and E. Bouali. „Reliability of an unstable conduit use for natural gas transportation", in ESREL 2006 - Safety and Reliability for Managing Risk Conference, Estoril, Portugal, 2006.

[6] M. Hari Prasad, G. Rami Reddy, A. Srividya and A.K. Verma. „Applying mechanistic models to reliability evaluation of mechanical components - an illustra- 
tion", in Annals of Nuclear Energy, vol. 38(7), July 2011, pp. 1447-1451.

[7] T.A. Hendrickson, J.S. Leonard and D.A. Weise. „Application of magnetic bearing technology for vibration free rotating machinery", in Naval Engineers Journal, vol. 99(3), March 2009, pp. 107-111.

[8] J.L. Cook and J.E. Ramirez-Marquez. „Reliability analysis of cluster-based ad-hoc networks", in Reliability Engineering \& System Safety, vol. 93(10), October 2008, pp. 1512-1522.

[9] J.Vance, F. Zeidan and B. Murphy. „Bearings and their effect on rotordynamics", in Machinery vibration and rotordynamics, vol. 1(1), May 2010, pp. 171-269.

Belhadef Rachid

Faculty of Science and Technology,

University of Sedik Ben yahia of Jijel, ALGERIA

e-mail: r.belhadef@univ-biskra.dz

Ahmed Hafaifa

Applied Automation and Industrial Diagnostics Laboratory,

University of Djelfa 17000 DZ, ALGERIA

e-mail: hafaifa.ahmed.dz@ieee.org

Mohamed Boumehraz

Faculty of Science and Technology

University of Biskra 7000 DZ, ALGERIA

e-mail: m.boumehraz@univ-biskra.dz
[10] G. Levitin. Computational intelligence in reliability engineering: Evolutionary techniques in reliability analysis and optimization (Series: Studies in Computational Intelligence). vol. 39, Berlin: Springer-Verlag, 2007.

[11] Ming Chen H., Principles of bearing design. in Compressor Handbook, chapter 19, ed. Hanlon P.C., McGraw-Hill, 2001.

[12] M. Guemana, S. Aissani and A. Hafaifa. „Use a new calibration method for gas pipelines: An advanced method improves calibrating orifice flowmeters while reducing maintenance costs", in Hydrocarbon Processing Journal, vol. 90(8), August 2011, pp. 63-70. 